\title{
Erratum to: Experience with combination of cisplatin plus gemcitabine chemotherapy and intensity-modulated radiotherapy for locoregionally advanced nasopharyngeal carcinoma
}

Xiayun He - Dan Ou - Hongmei Ying - Guopei Zhu •

Chaosu Hu $\cdot$ Taifu Liu

Published online: 5 August 2011

(C) Springer-Verlag 2011

Erratum to: Eur Arch Otorhinolaryngol

DOI 10.1007/s00405-011-1669-9

Unfortunately, the family name of the first author in the online published article is swapped. The correct first name is Xiayun and the last name is He.

The online version of the original article can be found under doi:10.1007/s00405-011-1669-9.

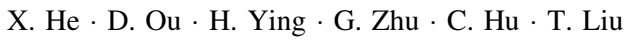
Department of Radiation Oncology, Fudan University Shanghai Cancer Center, 200032 Shanghai, China

X. He - D. Ou $\cdot$ H. Ying $\cdot$ G. Zhu $\cdot$ C. Hu $(\bowtie) \cdot$ T. Liu Department of Oncology, Shanghai Medical College, Fudan University, 399 Ling Ling Road, 200032 Shanghai, China

e-mail: chaosuhush@yahoo.cn 